Pacific Journal of Mathematics

A TRANSPLANTATION THEOREM FOR ULTRASPHERICAL 


\section{A TRANSPLANTATION THEOREM FOR ULTRASPHERICAL COEFFICIENTS}

\section{RichaRd ASkey aND Stephen WaINGeR}

Let $f(\theta)$ be integrable on $(0, \pi)$ and define

$$
a_{n}=\int_{0}^{\pi} f(\theta) \cos n \theta d \theta, \quad b_{n}=n^{1 / 2} \int_{0}^{\pi} f(\theta) P_{n}(\cos \theta)(\sin \theta)^{1 / 2} d \theta
$$

where $P_{n}(x)$ is the Legendre polynomial of degree $n$. Then

$$
c \leqq \sum_{n=0}^{\infty}\left|a_{n}\right|^{p}(n+1)^{\infty} / \sum_{n=0}^{\infty}\left|b_{n}\right|^{p}(n+1)^{\infty} \leqq C
$$

for $1<p<\infty,-1<\alpha<p-1$, where $C$ and $c$ depend on $p$ and $\alpha$ but not on $f$. From this we obtain a form of the Marcinkiewicz multiplier theorem for Legendre coefficients. Also an analogue of the Hardy-Littlewood theorem on Fourier coefficients of monotone coefficients is obtained. In fact, any norm theorem for Fourier functions can be transplanted by (1) to a corresponding theorem for Legendre coefficients.

Actually, the main theorem of this paper deals with ultraspherical coefficients and (1) is just a typical special case, which is stated as above for simplicity.

Let $P_{n}^{\lambda}(x)$ be defined by $\left(1-2 r x+r^{2}\right)^{-\lambda}=\sum_{n=0}^{\infty} P_{n}^{\lambda}(x) r^{n}$ for $\lambda>0$. The functions $P_{n}^{\lambda}(\cos \theta)$ are orthogonal on $(0, \pi)$ with respect to the measure $(\sin \theta)^{2 \lambda} d \theta$ and

$$
\int_{0}^{\pi}\left[P_{n}^{\lambda}(\cos \theta)\right]^{2}(\sin \theta)^{2 \lambda} d \theta=\frac{\Gamma(n+2 \lambda) \Gamma(1 / 2) \Gamma(\lambda+1 / 2)}{n !(n+\lambda) \Gamma(\lambda) \Gamma(2 \lambda)}=\left[t_{n}^{\lambda}\right]^{-2} .
$$

Observe that $t_{n}^{\lambda}=A n^{1-\lambda}+O\left(n^{-\lambda}\right)$ where $A$ will denote a constant whose numerical value is of no interest to us. For simplicity we set $\varphi_{n}^{\lambda}(\theta)=$ $t_{n}^{\lambda} P_{n}^{\lambda}(\cos \theta)(\sin \theta)^{\lambda}$. The functions $\left\{\varphi_{n}^{\lambda}(\theta)\right\}_{n=0}^{\infty}$ form a complete orthonormal sequence of functions on $(0, \pi)$ which for $\lambda=1$ reduce to $\{A \sin (n+1) \theta\}_{0}^{\infty}$. Also $\lim _{\lambda \rightarrow 0} \varphi_{n}^{\lambda}(\theta)=A \cos n \theta$ so the functions $\varphi_{n}^{\lambda}(\theta)$ are generalizations of the trigonometric functions which are used in classical Fourier series. For uniformity we define $\varphi_{n}^{0}(\theta)=(2 / \pi)^{1 / 2} \cos n \theta$. Later we shall state an asymptotic formula for $\varphi_{n}^{\lambda}(\theta)$ which shows another close connection with trigonometric functions. In essence it says that $\phi_{n}^{\lambda}(\theta)$ looks like $\cos [(n+\lambda) \theta-\pi(\lambda / 2)]$. All of the facts about $\varphi_{n}^{\lambda}$ that are quoted without reference are in [15]. Since $\varphi_{n}^{\lambda}(\theta)$ are a bounded orthonormal sequence we may consider their Fourier coefficients. Let $f \in L^{1}(0, \pi)$ and define 


$$
a_{n}^{\lambda}=\int_{0}^{\pi} f(\theta) \varphi_{n}^{\lambda}(\theta) d \theta .
$$

Let $\left\|a_{n}\right\|_{p}=\left[\sum_{n=0}^{\infty}\left|a_{n}\right|^{p}\right]^{1 / p}$. Then using M. Riesz's inequality [12] for $b_{n}=\sum_{k \neq n} a_{k} /(n-k)$, i.e. $\left\|b_{n}\right\|_{p} \leqq A_{p}\left\|a_{n}\right\|_{p}, 1<p<\infty$, and Hilbert's inequality, i.e. if $c_{n}=\Sigma a_{k} /(n+k)$ then $\left\|c_{n}\right\|_{p} \leqq A_{p}\left\|a_{n}\right\|_{p}, 1<p<\infty$, it is easy to show that $\left\|a_{n}^{1}\right\|_{p} \leqq A_{p}\left\|a_{n}^{0}\right\|$ and conversely $\left\|a_{n}^{0}\right\|_{p} \leqq A_{p}\left\|a_{n}^{1}\right\|_{p}$, $1<p<\infty$. It is this inequality that we generalize to all $\lambda>0$. For some of the applications we actually want a slight generalization of the above. Instead of considering the $l^{p}$ norm we work in a weighted $l^{p}$ norm,

$$
\left\|a_{n}\right\|_{p, \alpha}=\left[\sum_{n=0}^{\infty}\left|a_{n}\right|^{p}(n+1)^{\alpha}\right]^{1 / p} .
$$

These applications will be given in the last section.

Our main theorem is as follows.

THEOREM 1. Let $f \in L^{1}(0, \pi)$ and define $a_{n}^{\lambda}$ as above. Then if $\left\|a_{n}\right\|_{p, \alpha}$ is defined by (2) we have

$$
A \leqq\left\|a_{n}^{\lambda}\right\|_{p, \alpha}\left\|a_{n}^{\mu}\right\|_{p, \alpha} \leqq A
$$

for all $\lambda, \mu \geqq 0$ and $1<p<\infty,-1<\alpha<p-1$.

It will be sufficient to prove the inequalities (3) when $\mu<\lambda<\mu+1$. We first give in detail the proof when $\mu=0$ and $0<\lambda<1$. The formulas that we use in this case are all in the literature and are reasonably well known. Also this proof is easier to follow than the proof of the general case. Then we will sketch the proof for general $\lambda, \mu, \mu<\lambda<\mu+1$. For simplicity we set $a_{n}^{0}=a_{n}$ and use $\cos n \theta$ instead of $\varphi_{n}^{0}(\theta)$.

Let $f_{r}(\theta)=\sum_{n=0}^{\infty} a_{n} r^{n} \cos n \theta$. Since $f_{r}(\theta) \rightarrow f(\theta)$ almost everywhere and boundedly in $L^{1}$ we have

$$
\begin{aligned}
a_{n}^{\lambda} & =\lim _{r \rightarrow 1} \int_{0}^{\pi} f_{r}(\theta) \varphi_{n}^{\lambda}(\theta) d \theta=\lim _{r \rightarrow 1} t_{n}^{\lambda} \int_{0}^{\pi} f_{r}(\theta) P_{n}^{\lambda}(\cos \theta)(\sin \theta)^{\lambda} d \theta \\
& =\lim _{r \rightarrow 1} \sum_{k=0}^{\infty} a_{k} r^{k} t_{n}^{\lambda} \int_{0}^{\pi} P_{n}^{\lambda}(\cos \theta) \cos k \theta(\sin \theta)^{\lambda} d \theta .
\end{aligned}
$$

We break the sum up into three parts, $0 \leqq k \leqq[n / 2],[n / 2]<k<2 n$ and $2 n \leqq k$. What we need in each of these intervals is a good estimate for $t_{n}^{\lambda} \int_{0}^{\pi} P_{n}^{\lambda}(\cos \theta) \cos k \theta(\sin \theta)^{\lambda} d \theta=G(k, n)$.

Consider first the case $k \geqq 2 n$. We use the following well-known representation for $P_{n}^{\lambda}(\cos \theta)$ in terms of $\cos j \theta$.

$$
P_{n}^{\lambda}(\cos \theta)=\sum_{j=0}^{n} \alpha_{j} \alpha_{n-j} \cos (n-2 j) \theta
$$


where $\alpha_{j}=(j)_{\lambda} / j !=A j^{\lambda-1}+O\left(j^{\lambda-2}\right)$. Then

$$
\begin{aligned}
G(k, n)= & \sum_{j=0}^{n} \alpha_{j} \alpha_{n-j} t_{n}^{\lambda} \int_{0}^{\pi}(\sin \theta)^{\lambda} \\
& \times[\cos (k-n+2 j) \theta+\cos (k+n-2 j) \theta] d \theta .
\end{aligned}
$$

Since $\int_{0}^{\pi}(\sin \theta)^{\lambda} \cos r \theta d \theta=O\left(r^{-1-\lambda}\right)$ and $k \geqq 2 n$ we see that

$$
\begin{aligned}
|G(k, n)| & \leqq A \sum_{j=1}^{n-1} j^{\lambda-1}(n-j)^{\lambda-1} k^{-1-\lambda} n^{1-\lambda} \\
& =O\left((n / k)^{\lambda} k^{-1}\right)=O\left(k^{-1}\right) .
\end{aligned}
$$

For the theorem that we want the last estimate $O\left(k^{-1}\right)$ is sufficient. Observe however that we actually have a better estimate. Because of this it is possible to change Theorem 1 to get similar theorems where the Fourier coefficients are defined by $\int_{0}^{\pi} f(\theta) P_{n}^{\lambda}(\cos \theta)(\sin \theta)^{\lambda} n^{\alpha} d \theta$ for various values of $\alpha$. A possible transplantation then goes to $\int_{0}^{\pi} f(\theta) P_{n}^{\lambda+\beta}(\cos \theta)(\sin \theta)^{\lambda+\beta} n^{\alpha-\beta} d \theta$. Or the $(\sin \theta)^{\lambda}$ can be omitted from both of these integrals. We mention these facts only because in the dual case different transplantation theorems have been considered by Muckenhoupt and Stein [11] and by the authors [3]. The reason that both types of theorems are true is best seen in the proof of the present theorem, which is essentially easier than either of the theorems in [11] or [3].

Next consider $G(k, n)$ for $k \leqq[n / 2]$. This time we need a formula of Szegö. For $0<\lambda<1$

$$
(\sin \theta)^{2 \lambda-1} P_{n}^{\lambda}(\cos \theta)
$$

$$
=\frac{2^{2-2 \lambda}}{\Gamma(\lambda)} \frac{\Gamma(n+2 \lambda)}{\Gamma(n+\lambda+1)} \sum_{j=0}^{\infty} f_{j, n}^{\lambda} \sin (n+2 j+1) \theta
$$

where $f_{0, n}^{\lambda}=1$ and

$$
f_{j, n}^{\lambda}=\frac{(1-\lambda)(2-\lambda) \cdots(j-\lambda)}{j !} \frac{(n+1) \cdots(n+j)}{(n+\lambda+1) \cdots(n+\lambda+j)} .
$$

See $[15$, p. 96]. A simple estimate shows that

$$
f_{j, n}^{\lambda}=O\left(j^{-\lambda}(n+j)^{-\lambda} n^{\lambda}\right) \text {. }
$$

Then

$$
\begin{aligned}
G(k, n)= & O\left[\sum_{j=0}^{\infty} n^{1-\lambda} f_{j, n}^{\lambda} n^{\lambda-1} \int_{0}^{\pi}(\sin \theta)^{1-\lambda}\right. \\
& \times[\sin (n+2 j-k+1) \theta+\sin (n+2 j+k+1) \theta] d \theta] \\
= & O\left[\sum_{j=0}^{\infty} f_{j, n}^{\lambda}(n+j)^{-2+\lambda}\right] \\
= & O\left(n^{-2+\lambda}\right)+O\left(\sum_{j=1}^{\infty} j^{-\lambda}(n+j)^{-2} n^{\lambda}\right)=O\left(n^{-1}\right) .
\end{aligned}
$$


As usual in results of this nature the region where $k$ and $n$ overlap is harder to handle. This is because a Hilbert transform of some sort always seems to arise. This time we not only have the usual Hilbert transform but we also get a strange variant of it. The transformation we encounter is

$$
b_{n}=\frac{1}{n} \sum_{k=[n / 2]}^{2 n} a_{k} \log \frac{n}{|n-k+\lambda+1|} .
$$

In $\S 2$ we prove the following lemma, which we will use in the following argument.

LEMMA 1. If $\left\{a_{n}\right\} \in l^{p, \alpha}, 1<p<\infty,-1<\alpha<p-1$, and

$$
b_{n}=\frac{1}{n} \sum_{k=[n / 2]}^{2 n} a_{k} \log \frac{n}{|n-k+\lambda+1|}
$$

then $\left\|b_{n}\right\|_{p, \alpha} \leqq A_{p}\left\|a_{n}\right\|_{p, \alpha}$.

For reference we state a form of the asymptotic formula for $P_{n}^{\lambda}(\cos \theta)$ which we will use, [15, p. 195].

For $0<\lambda<1,1 / n \leqq \theta \leqq \pi / 2$

$$
\begin{aligned}
P_{u}^{\lambda}(\cos \theta) & =\frac{\Gamma(n+2 \lambda)}{\Gamma(n+\lambda+1)}\left[\frac{A \cos \left\{(n+\lambda) \theta-\frac{\lambda \pi}{2}\right\}}{(\sin \theta)^{\lambda}}\right. \\
+ & \left.\frac{B \cos \left\{(n+\lambda+1) \theta-(\lambda+1) \frac{\pi}{2}\right\}}{(n+\lambda+1)(\sin \theta)^{\lambda+1}}+O\left(n^{-2}(\sin \theta)^{-\lambda-2}\right)\right] .
\end{aligned}
$$

where $A$ and $B$ depend upon $\lambda$ but not on $n$.

From this we have

$$
t_{n}^{\lambda} P_{n}^{\lambda}(\cos \theta)(\sin \theta)^{\lambda}=A \cos \left\{(n+\lambda) \theta-\frac{\lambda \pi}{2}\right\}
$$

$$
+\frac{B \cos \left\{(n+\lambda+1) \theta(\lambda+1) \frac{\pi}{2}\right\}}{n \sin \theta}+O\left((n \theta)^{-2}\right)+O\left(n^{-1}\right),
$$

where $1 / n \leqq \theta \leqq \pi / 2$ and the $O$ terms are uniform in $n$ and $\theta$. Also we shall use the fact that $t_{n}^{\lambda} P_{n}^{\lambda}(\cos \theta)(\sin \theta)^{\lambda}$ are uniformly bounded functions, $[15,7.33,6]$. Instead of considering

$$
t_{n}^{\lambda} \int_{0}^{\pi} P_{n}^{\lambda}(\cos \theta)(\sin \theta)^{\lambda} \cos k \theta d \theta
$$

we may consider $\int_{0}^{\pi / 2}$ since the integrand is either even or odd with respect to $\theta=\pi / 2$. Using (7) we get 


$$
\begin{aligned}
t_{n}^{\lambda} \int_{0}^{\pi / 2} P_{n}^{\lambda}(\cos \theta) & (\sin \theta)^{\lambda} \cos k \theta d \theta \\
= & t_{n}^{\lambda} \int_{1 / n}^{\pi / 2} P_{n}^{\lambda}(\cos \theta)(\sin \theta)^{\lambda} \cos k \theta d \theta+O\left(\frac{1}{n}\right) \\
= & A \int_{1 / n}^{\pi / 2} \cos \left\{(n+\lambda) \theta-\frac{\lambda}{2}\right\} \cos k \theta d \theta \\
& +B \int_{1 / n}^{\pi / 2} \frac{\cos \left\{(n+\lambda+1) \theta-(\lambda+1) \frac{\pi}{2}\right\} \cos k \theta d \theta}{n \sin \theta} \\
& +O\left[\int_{1 / n}^{\pi / 2} \frac{d \theta}{n^{2} \theta^{2}}\right]+O\left(\frac{1}{n}\right) \cdot
\end{aligned}
$$

The last two terms are $O(1 / n)$ and the first is $A^{\prime} /(n-k+\lambda)+O(1 / n)$. We need to consider the second term. Using the addition theorem for $\cos \theta$ we get $B / n \int_{1 / n}^{\pi / 2}[\{\cos (n-k+1) \theta\} / \sin \theta] d \theta+$ three more terms which are similar but easier to handle. Since $1 / \sin \theta-1 / \theta$ is a bounded function for $0<\theta \leqq \pi / 2$ we may instead consider

$$
J=\frac{B}{n} \int_{1 / n}^{\pi / 2} \frac{\cos (n-k+\lambda+1) \theta}{\theta} d \theta .
$$

Assume first that $k<n+1+\lambda$. Then changing variables by $(n-k+\lambda+1) \theta=y$, we find

$$
J=\frac{B}{n} \int_{(n-k+\lambda+1) / n}^{1} \frac{\cos y d y}{y}+\frac{B}{n} \int_{1}^{n-k+\lambda+1} \frac{\cos y d y}{y} .
$$

The second term is $O(1 / n)$ by an integration by parts. The first term is

$$
\begin{aligned}
\frac{B}{n} \int_{(n-k+\lambda+1) / n}^{1} \frac{d y}{y} & +O\left(\frac{1}{n} \int_{(n-k+\lambda+1) / n}^{1} y d y\right) \\
& =\frac{B}{n} \log \frac{n}{n-k+\lambda+1}+O\left(\frac{1}{n}\right) .
\end{aligned}
$$

If $k>n+\lambda+1$ we get instead that

$$
J=\frac{B}{n} \log \frac{n}{k-n-\lambda-1}+O\left(\frac{1}{n}\right) .
$$

Using all of the estimates, we have

$$
\begin{aligned}
a_{n}^{\lambda}= & O\left[\frac{1}{n} \sum_{k=0}^{[n / 2]}\left|a_{k}\right|\right]+A \sum_{k=[n / 2]}^{2 n} \frac{a_{k}}{n-k+\lambda} \\
& +\frac{B}{n} \sum_{k=[n / 2]}^{2 n} a_{k} \log \frac{n}{|n-k+\lambda+1|} \\
& +O\left[\frac{1}{n} \sum_{k=[n / 2]}^{2 n}\left|a_{k}\right|\right]+\lim _{r \rightarrow 1} \sum_{k=2 n}^{\infty} a_{k} r^{k} A(k, n)
\end{aligned}
$$


where $A(k, n)=O\left(k^{-1}\right)$.

To show the $l^{p, a}$ boundedness of these sums we need two forms of Hardy's inequality and M. Riesz's inequality for the discrete Hilbert transform as well as Lemma 1. The relevant forms of Hardy's inequality are in [6], p. 255, \#346 (a), (b), part $(\alpha)$. The continuous analogue of the $l^{p, \alpha}$ boundedness of the discrete Hilbert transform is in [5].

Using these inequalities we see that the first and fourth terms are bounded by Hardy's inequality. By dominated convergence we may let $r \rightarrow 1$ in the fifth term and it is bounded in $l^{p, \alpha}$ by Hardy's inequality. The second term is just the discrete Hilbert transform plus two terms like the first and last terms. Thus it is bounded in $l^{p, \alpha}$. The third term is handled by Lemma 1.

In actual fact the second and third terms given above are not exactly right since the terms in which $k$ and $n$ have opposite parity are zero. The notation to include this is too cumbersome to be worth including and this point causes no trouble.

To show that $\left\|a_{n}\right\|_{p, \alpha} \leqq A\left\|a_{n}^{\lambda}\right\|_{n, \alpha}$ observe that (formally)

$$
a_{k}=\int_{0}^{\pi} f(\theta) \cos k \theta d \theta=\sum_{n=0}^{\infty} a_{n}^{\lambda} t_{n}^{\lambda} \int_{0}^{\pi} P_{n}^{\lambda}(\cos \theta) \cos k \theta(\sin \theta)^{\lambda} d \theta .
$$

We have the same $G(k, n)$ that we analysed above and so no more work need be done on it. However there is the problem of Abel summability of ultraspherical expansions. Estimates for the Poisson kernel which allows us to prove the dominated $L^{1}$ convergence of the Abel means are in $[11, \S 4]$. The argument that is needed to prove this is well known.

We now consider the general case of Theorem 1 with $\mu<\lambda<\mu+1$. The proof proceeds along the same general lines but the formulas for $P_{n}^{\lambda}$ that we need are considerably more complicated. To take the place of (4) we need the following result of Gegenbauer [4].

If $0<\alpha<\beta$ then

$$
P_{n}^{\beta}(\cos \theta)=\sum_{j=0}^{[n / 2]} \alpha_{j} P_{n-2 j}^{a}(\cos \theta)
$$

where

$$
\alpha_{j}=\frac{\Gamma(\alpha)(n-2 j+\alpha) \Gamma(j+\beta-\alpha) \Gamma(n-j+\beta)}{\Gamma(\beta) \Gamma(\beta-\alpha) j ! \Gamma(n-j+\alpha+1)} .
$$

Instead of (5) we need a result that follows from (8) and is given in [2]. If $(\beta-1) / 2<\alpha<\beta$ then

$$
(\sin \theta)^{2 \alpha} P_{n}^{\alpha}(\cos \theta)=\sum_{j=0}^{\infty} \beta_{j} P_{n+2 j}^{\beta}(\cos \theta)(\sin \theta)^{2 \beta}
$$


where

$$
\beta_{j}=\frac{\Gamma(\beta) 2^{2 \beta-2 \alpha}(n+2 j) !(n+2 j+\beta) \Gamma(n+2 \alpha) \Gamma(n+j+\beta) \Gamma(j+\beta-\alpha)}{\Gamma(\beta-\alpha) \Gamma(\alpha) j ! n ! \Gamma(n+j+\alpha+1) \Gamma(n+2 j+2 \beta)} .
$$

Observe that $\beta_{j}$ is positive if $\alpha<\beta$. This result also holds for $\alpha>\beta$ but then the coefficients are no longer positive and changes must be made for $\alpha=\beta+1, \beta+2, \cdots$, since the right hand side is then a finite sum. A simple computation shows that

$$
\alpha_{j} \sim(n-2 j+\alpha) j^{\beta-\alpha-1} n^{\beta-\alpha-1}
$$

and

$$
\beta_{j} \sim(n+2 j)^{2-2 \beta} n^{2 \alpha-1} j^{\beta-\alpha-1}(n+j)^{\beta-\alpha-1} \sim(n+j)^{1-\beta-\alpha} n^{2 \alpha-1} j^{\beta-\alpha-1},
$$

for $\alpha<\beta$. For $\alpha>\beta$ and $\alpha \neq \beta+1, \beta+2, \cdots$, we have

$$
\left|\beta_{j}\right| \sim(n+j)^{1-\beta-\alpha} n^{2 \alpha-1} j^{\beta-\alpha-1} \text {. }
$$

By $a_{j} \sim b_{j}$ we mean $0<c \leqq a_{j} / b_{j} \leqq C<\infty$.

If in (9) we let $n=0$ and use (1) we have

$$
\left|\int_{0}^{\pi}(\sin \theta)^{2 \alpha} P_{2 j}^{\beta}(\cos \theta) d \theta\right|=O\left(j^{2 \beta-2 \alpha-2}\right)
$$

Next we need something to take the place of $\cos x \cos y=$ $[\cos (x+y)+\cos (x-y)] / 2$ and $\sin x \cos y=[\sin (x+y)+\sin (x-y)] / 2$. For the first we use a formula of Dougall which is given in [9] and reduces to it for $\lambda \rightarrow 0$. If $\lambda>0$ then

$$
\frac{P_{n}^{\lambda}(x)}{P_{n}^{\lambda}(1)} \frac{P_{m}^{\lambda}(x)}{P_{m}^{\lambda}(1)}=\sum_{k=|n-m|}^{n+m} c_{\lambda}(k, m, n) \frac{P_{k}^{\lambda}(x)}{P_{k}^{\lambda}(1)}
$$

where $c_{\lambda}(k, m, n) \geqq 0$ and $\sum_{k} c_{\lambda}(k, m, n)=1$. We define $c_{\lambda}(k, m, n)=0$ if $k<|n-m|$ or $k>n+m$ and then we may sum on all nonnegative $k$. The numbers $c_{\lambda}$ are known [9], but we shall not need them in our argument.

For the second formula above we use the following substitute which again reduces to it for $\lambda \rightarrow 0$. If $\lambda>0$ then

$$
\frac{P_{n}^{\lambda+1}(x)}{P_{n}^{\lambda+1}(1)} \frac{P_{m}^{\lambda}(x)}{P_{m}^{\lambda}(1)}=\sum_{k=|n-m|-2}^{n+m} d_{\lambda}(k, m, n) \frac{P_{k}^{\lambda+1}(x)}{P_{k}^{\lambda+1}(1)}
$$

where $d_{\lambda} \geqq 0$ if $n \geqq m-1$. This is found in [1]. From (14) it follows that $\sum_{k=0}^{\infty} d_{\lambda}(k, m, n)=1$ where $d_{\lambda}(k, m, n)=0$ if $k<|n-m|-2$ or $k>n+m$. Finally recall that

$$
P_{n}^{\lambda}(1) \sim n^{2 \lambda-1}
$$


These results are sufficient to allow us to estimate $\int_{0}^{\pi} \varphi_{n}^{\lambda}(\theta) \varphi_{k}^{\mu}(\theta) d \theta$ for $\mu<\lambda<\mu+1$ and $k \leqq n / 2$ or $n \leqq k / 2$. To estimate this integral for $k / 2<n<2 k$ we use the following asymptotic formulas due to Szegö.

Lemma 2. Let $\mu>0, \mu$ not an integer. Then

$$
\begin{aligned}
& P_{n}^{\mu}(\cos \theta)=\frac{2}{\pi} \sin \pi \mu \frac{\Gamma(n+2 \mu)}{\Gamma(\mu)} \\
& \quad \times\left[\sum_{m=0}^{p-1} \frac{\Gamma(m+\mu) \Gamma(m-\mu+1)}{\Gamma(n+m+\mu+1) m !} \frac{\cos \left[(n+m+\mu) \theta-(m+\mu) \frac{\pi}{2}\right]}{(2 \sin \theta)^{m+\mu}}\right]+R_{p}
\end{aligned}
$$

where

$$
\left|R_{p}\right|=O\left[(\sin \theta)^{-p-\mu} n^{\mu-p_{-1}}\right]
$$

and the $O$ holds uniformly for $0<\theta<\pi$.

For $\mu=1,2,3, \cdots$ we have

LEMMA 3.

$$
\begin{aligned}
P_{n}^{\mu}(\cos \theta)= & 2 \sum_{m=0}^{\mu-1}(-1)^{m}\left(\begin{array}{c}
m+\mu-1 \\
m
\end{array}\right)\left(\begin{array}{c}
n+2 \mu-1 \\
\mu-m+1
\end{array}\right) \\
& \times \frac{\cos \left[(n+m+\mu) \theta-(m+\mu) \frac{\pi}{2}\right]}{[(2 \sin \theta)(m+\mu)]} .
\end{aligned}
$$

The same estimates hold for an error term in Lemma 3 as in Lemma 2 if one stops before $m=\mu-1$. These two lemmas are in $[14$, p. 49 and p. 59]. In fact we do not need the full force of either of these Lemmas but they are relatively inaccessible and not as well known as they should be.

Now to complete the proof of Theorem 1. Let $\mu<\lambda<\mu+1$ and $f_{r}(\theta)=\sum_{n=0}^{\infty} a_{n}^{\mu} r^{n} \varphi_{n}^{\mu}(\theta)$. Then by dominated convergence and the boundedness of the Abel means of an ultraspherical expansion we have

$$
a_{n}^{\lambda}=\lim _{r \rightarrow 1^{-}} \int_{0}^{\pi} f_{r}(\theta) \varphi_{n}^{\lambda}(\theta) d \theta=\lim _{r \rightarrow 1^{-}} \sum_{k=0}^{\infty} \alpha_{k}^{\mu} r^{k} \int_{0}^{\pi} \varphi_{k}^{\mu}(\theta) \varphi_{n}^{\lambda}(\theta) d \theta
$$

As above we need to estimate $\int_{0}^{\pi} \varphi_{k}^{\lambda}(\theta) \varphi_{n}^{\mu}(\theta) d \theta=G(k, n)$ for three cases, $k \leqq n / 2, n / 2<k<2 n$, and $2 n \leqq k$. Consider the third case first. Using (8) and (13) we have 


$$
\begin{aligned}
G(k, n)= & t_{n}^{\lambda} t_{k}^{\mu} \int_{0}^{\pi} P_{k}^{\mu}(\cos \theta) P_{n}^{\lambda}(\cos \theta)(\sin \theta)^{\lambda+\mu} d \theta \\
= & \sum_{j=0}^{[n / 2]} t_{n}^{\lambda} t_{k}^{\mu} \alpha_{j} \int_{0}^{\pi} P_{k}^{\mu}(\cos \theta) P_{n-2 j}^{\mu}(\cos \theta)(\sin \theta)^{\lambda+\mu} d \theta \\
= & \sum_{j=0}^{[n / 2]} t_{n}^{\lambda} t_{k}^{\mu} \alpha_{j} P_{k}^{\mu}(1) P_{n-2 j}^{\mu}(1) \sum_{l=0}^{\infty} c_{\mu}(l, k, n-2 j)\left[P_{l}^{\mu}(1)\right]^{-1} \\
& \cdot \int_{0}^{\pi} P_{l}^{\mu}(\cos \theta)(\sin \theta)^{\lambda-\mu}(\sin \theta)^{2 \mu} d \theta
\end{aligned}
$$

Then using (10), (12), (15), and recalling that $c_{\mu}(l, k, n-2 j)=0$ unless $k-n+2 j \leqq l \leqq k+n-2 j$ and so $l \sim k$.

For simplification of printing we use $n, k, j$ in the following arguments instead of $n+1, k+1, j+1$, etc. This leads to some infinite terms which clearly aren't infinite and they are to be interpreted in the obvious way.

$$
\begin{aligned}
|G(k, n)| & \leqq \sum_{j=0}^{[n / 2]}(n)^{1-\lambda}(k)^{1-\mu}(n-2 j+\mu)(j)^{\lambda-\mu-1}(n)^{\lambda-\mu-1}(n-2 j)^{2 \mu-1}(k)^{\mu-\lambda-2} \\
& \leqq A(n / k)^{\lambda}(k)^{-1} \leqq A(k)^{-1}
\end{aligned}
$$

Next we consider $G(k, n)$ for $k \leqq n / 2$. Using (9) and (14) we have

$$
\begin{aligned}
G(k, n)= & \sum_{j=0}^{\infty} t_{n}^{\lambda} t_{k}^{\mu} \beta_{j} \int_{0}^{\pi} P_{k}^{\mu}(\cos \theta) P_{n+2 j}^{\mu+1}(\cos \theta)(\sin \theta)^{3 \mu-\lambda+2} d \theta \\
= & \sum_{j=0}^{\infty} t_{n}^{\lambda} t_{k}^{\mu} \beta_{j} P_{k}^{\mu}(1) P_{n+2 j}^{\mu+1}(1) \sum_{l=0}^{\infty} d_{\mu}(l, k, n+2 j)\left[P_{l}^{\mu+1}(1)\right]^{-1} \\
& \cdot \int_{0}^{\pi} P_{l}^{\mu+1}(\cos \theta)(\sin \theta)^{\mu-\lambda}(\sin \theta)^{2 \mu+2} d \theta
\end{aligned}
$$

This time $d_{\lambda}(l, k, n+2 j)=0$ unless $n / 2+j \leqq l \leqq 2 n+4 j$ (actually it is zero for many values in this range also but that doesn't matter) and so $l \approx n+2 j$ and thus using (11), (12), and (15) we obtain

$$
\begin{aligned}
|G(k, n)| & \leqq \sum_{j=0}^{\infty}(n)^{1-\lambda}(k)^{1-\mu}(k)^{2 \mu-1} \beta_{j} \int_{0}^{\pi} P_{n+2 j}^{\mu+1}(\cos \theta)(\sin \theta)^{3 \mu-\lambda+2} d \theta \\
& \leqq \sum_{j=0}^{\infty}(n)^{1-\lambda}(k)^{\mu}(n)^{2 \lambda-1}(n+j)^{-\lambda-\mu}(j)^{\mu-\lambda} \int_{0}^{\pi} P_{n+2 j}^{\mu+1}(\cos \theta)(\sin \theta)^{3 \mu-\lambda+2} d \theta \\
& \leqq(n)^{\lambda}(k)^{\mu} \sum_{j=0}^{\infty}(j)^{\mu-\lambda}(n+j)^{-\lambda-\mu}(n+j)^{-2+2(\mu+1)-2+\lambda-3 \mu} \\
& \leqq(n)^{\lambda}(k)^{\mu}\left[\sum_{j=0}^{n}(j)^{\mu-\lambda} n^{-2-2 \mu}+\sum_{j=n}^{\infty}(j)^{-2-\mu-\lambda}\right] \\
& \leqq(n)^{\lambda}(k)^{\mu}\left[(n)^{-1-\mu-\lambda}+(n)^{-1-\mu-\lambda}\right] \leqq[k / n]^{\mu}(n)^{-1}
\end{aligned}
$$

For the terms with $k / 2 \leqq n \leqq 2 k$ we use Lemmas 2 and 3 . As in the case $\mu=0,0<\lambda<1$ we first reduce the integral to

$$
\int_{1 / n}^{\pi / 2} \varphi_{n}^{\lambda}(\theta) \varphi_{k}^{\mu}(\theta) d \theta+O\left(n^{-1}\right)
$$


and then terms of the same type as previously appear. The proof is then finished by the same appeal to Hardy's inequality, M. Riesz's inequality, and Lemma 1.

Theorem 1 then follows by a repeated application of the inequalities just proven.

2. A lemma. We now give a proof of Lemma 1. Recall that

$$
b_{n}=\frac{1}{n} \sum_{k=[n / 2]}^{2 n} a_{k} \log \frac{n}{|n+\lambda+1-k|} \text {. }
$$

We define $A_{k}=\sum_{j=[n / 2]}^{k} a_{j}$. Then

$$
\begin{aligned}
b_{n}= & \frac{1}{n} \sum_{k=[n / 2]+1}^{2 n}\left(A_{k}-A_{k-1}\right) \log \frac{n}{|n+\lambda+1-k|}+\frac{1}{n} a_{[n / 2]} \log \frac{n}{\frac{n}{2}+\lambda+1} \\
= & \frac{1}{n} \sum_{k=[n / 2]+1}^{2 n-1} A_{k}\left[\log \frac{n}{|n+\lambda+1-k|}-\log \frac{n}{|n+\lambda-k|}\right] \\
& +O\left(\frac{1}{n} a_{[n / 2]}\right)+O\left(\frac{A_{2 n}}{n^{2}}\right) \\
= & +\frac{1}{n} \sum_{k=[n / 2]}^{2 n} A_{k} \log \left|\frac{n+\lambda-k}{n+\lambda+1-k}\right|+R_{n}
\end{aligned}
$$

where $R_{n}$ is a bounded sequence in $l^{p, \alpha}$ if $\left\{a_{n}\right\} \in l^{p, \alpha}$. But

$$
\begin{aligned}
\log \left|\frac{n+\lambda-k}{n+\lambda+1-k}\right| & =-\log \left|1+\frac{1}{n+\lambda-k}\right| \\
& =+\frac{1}{k-n-\lambda}+O \frac{1}{(k-n-\lambda)^{3}} .
\end{aligned}
$$

So we have

$$
b_{n}=-\frac{1}{n} \sum_{k=[n / 2]}^{2 n} \frac{A_{k}}{n+\lambda-k}+\frac{1}{n} \sum_{k=[n / 2]}^{2 n} \frac{A_{k}}{(k-n-\lambda)^{2}}+R_{n} .
$$

The second term is a bounded sequence in $l^{p, \alpha}$ by $[6$, p. 198, \#274]. We write the first term as

$$
-\frac{1}{n} \sum_{k=[n / 2]}^{2 n} \frac{A_{k}}{n+\lambda-k}=\sum_{k=[n / 2]}^{2 n} \frac{\frac{-A_{k}}{(k-\lambda)}}{n+\lambda-k}+\sum_{k=[n / 2]}^{2 n} \frac{A_{k}}{n(k-\lambda)} .
$$

But $A_{k} /(k-\lambda)$ is in $l^{p, \alpha}$ and so we have that $\left\{b_{n}\right\}$ is an $l^{p, \alpha}$ sequence by Hardy's inequality and M. Riesz's inequality for the discrete Hilbert transform.

A similar proof also shows that 


$$
c_{n}=\sum_{k=[n] 2]}^{2 n} \frac{a_{k}}{k} \log \frac{n}{|n-k+\alpha|}
$$

is a bounded operator for $\alpha$ not an integer. If $\alpha$ is an integer the transformations are bounded if the term when the logarithm is undefined is dropped.

A similar theorem is also true in the continuous case where an integration by parts takes the place of our summation by parts.

3. Applications, Our first application is an analogue of a theorem of Hardy and Littlewood concerning the Fourier coefficients of even functions, monotonically decreasing in $(0, \pi),[16, \mathrm{p} .130]$. Their theorem is

THEOREM A.' If $f(\theta)$ is a decreasing integrable function on $(0, \pi)$ and if $a_{n}$ are the Fourier cosine coefficients of $f$, then

$$
\left[\sum_{n=0}^{\infty}\left|a_{n}\right|^{p}(n+1)^{\alpha}\right]^{1 / p}
$$

is finite if and only if

$$
\left[\int_{0}^{\pi}|f(\theta)|^{p} \theta^{p-2+\alpha} d \theta\right]^{1 / p}
$$

is finite, $1<p<\infty,-1<\alpha<p-1$.

From this and Theorem 1 we obtain

THEOREM 2. Let $f(\theta)$ be decreasing and integrable on $(0, \pi)$ and $a_{n}=t_{n}^{\lambda} \int_{0}^{\pi} f(\theta) P_{n}^{\lambda}(\cos \theta)(\sin \theta)^{\lambda} d \theta, \quad 0<\lambda$. Then $\left[\sum_{n=0}^{\infty}\left|a_{n}\right|^{p}(n+1)^{\alpha}\right]^{1 / p}$ is finite if and only if $\left[\int_{0}^{\pi}|f(\theta)|^{p} \theta^{p-2-\alpha} d \theta\right]^{1 / p}$ is finite, $1<p<\infty$, $-1<\alpha<p-1$

Another application is the analogue of the Marcinkiewicz Multiplier theorem. In the case of Fourier coefficients it is due to Sunouchi [13] for $\left\{a_{n}\right\} \in l^{p}$ and to Igari [10] for $\left\{a_{n}\right\} \in l^{p, \alpha}$.

Theorem B. Let $f(\theta) \in L^{1}(0, \pi), a_{n}=\int_{0}^{\pi} f(\theta) \cos n \theta d \theta,|t(\theta)| \leqq C$,

$$
\int_{\pi 2^{-n-1}}^{\pi 2^{-n}}|d t(\theta)| \leqq C, \quad n=0,1, \cdots
$$

Then if $b_{n}=\int_{0}^{\pi} t(\theta) f(\theta) \cos n \theta d \theta$ and $\left\{a_{n}\right\} \in l^{p, \alpha}, 1<p<\infty,-1<\alpha<p-1$, we have $\left\{b_{n}\right\} \in l^{p, \alpha}$ and $\left\|b_{n}\right\|_{p, \alpha} \leqq A\left\|a_{n}\right\|_{p, \alpha}$.

From this we get a form of the Marcinkiewicz theorem for ultra- 
spherical coefficients.

THEOREM 3. Let $f(\theta) \in L^{1}(0, \pi), \quad a_{n}=t_{n}^{\lambda} \int_{0}^{\pi} f(\theta) P_{n}^{\lambda}(\cos \theta)(\sin \theta)^{\lambda} d \theta$, $\lambda>0,|t(\theta)| \leqq C$,

$$
\int_{\pi 2^{-n-1}}^{\pi 2^{-n}}|d t(\theta)| \leqq C, \quad n=0,1, \cdots
$$

Then if $b_{n}=t_{n}^{\lambda} \int_{0}^{\pi} t(\theta) f(\theta) P_{n}^{\lambda}(\cos \theta)(\sin \theta)^{\lambda} d \theta$ and if $\left\{a_{n}\right\} \in l^{p, \alpha}, 1<p<\infty$, $-1<\alpha<p-1$ then $\left\{b_{n}\right\} \in l^{p, \alpha}$ and $\left\|b_{n}\right\|_{p, \alpha} \leqq A\left\|a_{n}\right\|_{p, \alpha}$.

For $p=2$ Hirschman has already obtained a form of the Marcinkiewicz theorem. If we let

$$
t_{r}(\theta)= \begin{cases}1 & 0 \leqq \theta \leqq 1 / r \\ 0 & 1 / r\end{cases}
$$

then we get the projection theorem of Hirschman [8] but only for ultraspherical coefficients. Hirschman proves his result for Jacobi coefficients and presumably Theorem 1 is also true for Jacobi polynomials. However this is still open.

\section{REFERENCES}

1. R. Askey, Products of ultraspherical polynomials, to appear in Amer. Math. Monthly.

2. - Orthogonal expansions with positive coefficients, to appear in Proc. Amer. Math. Soc.

3. R. Askey and S. Wainger, A transplantation theorem for ultraspherical series, to appear in the Ill. J. Math.

4. L. Gegenbauer, Zur Theorie der Functionen $C_{n}^{\nu}(x)$, Denkschriften der Akademie der Wissenschaften in Wien, Mathematischnaturwissenschaftliche Klasse, 48 (1884), 293316.

5. G. H. Hardy and J. E. Littlewood, Some theorems on Fourier series and Fourier power series, Duke Math. J. 2 (1936), 354-381.

6. G. H. Hardy, J. E. Littlewood, and G. Polya, Inequalities, Cambridge, 1952.

7. I. I. Hirschman, Jr., Weighted quadratic norms and ultraspherical polynomials, II, Trans. Amer. Math. Soc. 91 (1959), 314-329.

8. - Projections associated with Jacobi polynomials, Proc. Amer. Math. Soc. 8 (1957), 286-290.

9. H. Y. Hsü, Certain integrals and infinite series involving ultaspherical polynomials and Bessel functions, Duke Math. J. 4 (1938), 374-383.

10. S. Igari, On the decomposition theorems of Fourier transforms with weighted norms, Tohôku Math. J. (2) 15 (1963), 6-36.

11. B. Muckenhoupt and E. Stein, Classical expansions and their relation to conjugate harmonic functions, Trans. Amer. Math. Soc. 118 (1965), 17-92.

12. M. Riesz, Sur les fonctions conjuguees, Math. Z. 27 (1928), 218-244.

13. G. Sunouchi, Discrete analogue of a theorem of Littlewood-Paley, Tohôku Math.

J. (2) 13 (1961), 320-328. 
14. G. Szegö, Asymptostiche Entwicklungen der Jacobischen Polynome, Schriften der Konigsberger Gelehrten Gesellschaft, Naturwissen Schaftliche Klasse, 3 (1933).

15. - Orthogonal Polynomials, New York, 1959.

16. A. Zygmund, Trigonometric Series, vol. 2, (Cambridge), 1959.

UNIVERSITY OF WISCONSIN

CoRnell UNIVERSITY 



\section{PACIFIC JOURNAL OF MATHEMATICS}

\section{EDITORS}

\author{
H. SAMmLSON \\ Stanford University \\ Stanford, California \\ R. M. BLUMENTHAL \\ University of Washington \\ Seattle, Washington 98105
}

\author{
*J. DUGUNDJI \\ University of Southern California \\ Los Angeles, California 90007
}

RICHARD ARENS

University of California

Los Angeles, California 90024

\section{E. F. BECKENBACH \\ B. H. NEUMANN \\ ASSOCIATE EDITORS}

\section{SUPPORTING INSTITUTIONS}

\author{
UNIVERSITY OF BRITISH COLUMBIA \\ CALIFORNIA INSTITUTE OF TECHNOLOGY \\ UNIVERSITY OF CALIFORNIA \\ MONTANA STATE UNIVERSITY \\ UNIVERSITY OF NEVADA \\ NEW MEXICO STATE UNIVERSITY \\ OREGON STATE UNIVERSITY \\ UNIVERSITY OF OREGON \\ OSAKA UNIVERSITY \\ UNIVERSITY OF SOUTHERN CALIFORNIA
}

\author{
STANFORD UNIVERSITY \\ UNIVERSITY OF TOKYO \\ UNIVERSITY OF UTAH \\ WASHINGTON STATE UNIVERSITY \\ UNIVERSITY OF WASHINGTON \\ * * * \\ AMERICAN MATHEMATICAL SOCIETY \\ CHEVRON RESEARCH CORPORATION \\ TRW SYSTEMS \\ NAVAL ORDNANCE TEST STATION
}

\footnotetext{
Mathematical papers intended for publication in the Pacific Journal of Mathematics should be typewritten (double spaced). The first paragraph or two must be capable of being used separately as a synopsis of the entire paper. It should not contain references to the bibliography. Manu. scripts may be sent to any one of the four editors. All other communications to the editors should be addressed to the managing editor, Richard Arens at the University of California, Los Angeles, California 90024.

50 reprints per author of each article are furnished free of charge; additional copies may be obtained at cost in multiples of 50 .
}

The Pacific Journal of Mathematics is published monthly. Effective with Volume 16 the price per volume (3 numbers) is $\$ 8.00$; single issues, $\$ 3.00$. Special price for current issues to individual faculty members of supporting institutions and to individual members of the American Mathematical Society: $\$ 4.00$ per volume; single issues $\$ 1.50$. Back numbers are available.

Subscriptions, orders for back numbers, and changes of address should be sent to Pacific Journal of Mathematics, 103 Highland Boulevard, Berkeley 8, California.

Printed at Kokusai Bunken Insatsusha (International Academic Printing Co., Ltd.), No. 6, 2-chome, Fujimi-cho, Chiyoda-ku, Tokyo, Japan.

PUBLISHED BY PACIFIC JOURNAL OF MATHEMATICS, A NON-PROFIT CORPORATION

The Supporting Institutions listed above contribute to the cost of publication of this Journal, but they are not owners or publishers and have no responsibility for its content or policies.

* Paul A. White, Acting Editor until J. Dugundji returns. 


\section{Pacific Journal of Mathematics}

\section{Vol. 16, No. $3 \quad$ BadMonth, 1966}

Gert Einar Torsten Almkvist, Stability of linear differential equations with

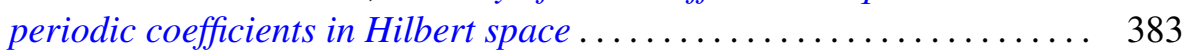

Richard Allen Askey and Stephen Wainger, A transplantation theorem for ultraspherical coefficients ................................ 393

Joseph Barback, Two notes on regressive isols .................. 407

Allen Richard Bernstein and Abraham Robinson, Solution of an invariant subspace problem of K. T. Smith and P. R. Halmos .............. 421

P. R. Halmos, Invariant subspaces of polynomially compact operators . . . . 433

Leon Bernstein, New infinite classes of periodic Jacobi-Perron algorithms.................................... 439

Richard Anthony Brualdi, Permanent of the direct product of matrices .... . 471

W. Wistar (William) Comfort and Kenneth Allen Ross, Pseudocompactness and uniform continuity in topological groups .................. 483

James Michael Gardner Fell, Algebras and fiber bundles . . . . . . . . . . . . 497

Alessandro Figà-Talamanca and Daniel Rider, A theorem of Littlewood and lacunary series for compact groups ..................... 505

David London, Two inequalities in nonnegative symmetric matrices...... 515

Norman Jay Pullman, Infinite products of substochastic matrices ........ 537

James McLean Sloss, Reflection and approximation by interpolation along

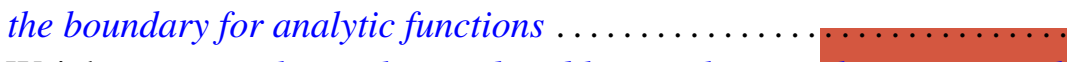

Carl Weinbaum, Visualizing the word problem, with an application to sixth groups................................... 